\title{
The relationship of dietary fish intake to level of pulmonary function in the first National Health and Nutrition Survey (NHANES I)
}

\author{
J. Schwartz*, S.T. Weiss**
}

The relationship of dietary fish intake to level of pulmonary function in the first National Health and Nutrition Survey (NHANES I). J. Schwartz, S.T. Weiss. (CERS Journals Ltd 1994.

ABSTRACT: Eicosapentaenoic acid (EPA), which predominates in marine fish, tends to counteract and inhibit the uptake and incorporation of arachidonic acid and membrane phospholipids and dilute arachidonic acid as a potential substrate for oxidation. Thus, fish intake may be protective for the occurrence of asthma and other pulmonary diseases.

We wanted to examine the relationship between the effect of chronic dietary intake to fish and its relationship to level of pulmonary function. We performed this analysis using data from the First National Health and Nutritional Examination Survey (NHANES I). A detailed subsample of 2,526 adults had a medical history questionnaire, that included a 24-hour dietary recall, and performed spirometric examination. Log of forced expiratory volume in one second $\left(\mathrm{FEV}_{1}\right)$ served as a dependent variable in regression analysis which included an adjustment for height, age, cigarette smoking and gender.

When added to the regression model including the above variables dietary fish intake showed a protective association with $\mathrm{FEV}_{1}(\beta=0.008 \pm 0.004, p=0.028)$. When smokers were excluded from the analysis, the effect of fish intake on pulmonary function appeared to increase slightly $(\beta=0.0108 \pm 0.006, p=0.61)$.

These data suggest that chronic dietary intake of fish is associated with higher levels of pulmonary function and is consistent with the hypothesis of an effect of fish oil on arachidonic acid metabolism.

Eur Respir J., 1994, 7, 1821-1824.
*Department of Environmental Health, Harvard School of Public Health. **Channing Laboratory, Department of Medicine, Brigham and Women's Hospital, Boston, MA and the Department of Pulmonary and Critical Care, Beth Israel Hospital, Boston, MA.

Correspondence: S.T. Weiss

Channing Laboratory

180 Longwood Avenue

Boston, MA

USA

Keywords: Arachidonic acid fish consumption lung function

Received: March 221993

Accepted after revision March 171994
Fatty acids are essential nutrients that are incorporated into a variety of cell membranes. These fatty acids, specifically linoleic, eicosapentaenoic and docosahexaenoic are found in varying amounts in the human diet and are metabolized to arachidonic acid. The further metabolism of arachidonic acid, by cyclooxygenase and lipoxygenase pathways, yields a variety of prostenoids and leukotrienes which are potentially toxic to cell membranes, smooth muscle, and epithelial and endothelial surfaces. In contrast, eicosapentaenoic acid (EPA), which predominates in marine fish, tends to counteract and inhibit the uptake and incorporation of arachidonic acid into membrane phospholipids and dilute arachidonic acid as a potential substrate for oxidization. The relatively low prevalences of coronary heart disease and asthma among Eskimos and other populations who have high dietary intake of EPA have suggested the hypothesis that fish intake may be protective for the occurrence of asthma and other diseases [1]. To date, no population based study has examined the effect of chronic dietary intake of fish and its relationship to level of pulmonary function. We chose to examine this hypothesis using dietary intake data from the First National Health and Nutrition Survey (NHANES I).

\section{Materials and methods}

The First National Health and Nutrition Examination Survey (NHANES I) was conducted at 90 locations across the United States between 1971 and 1975 [2]. The initial survey was collected in 1971-1974. An augmentation survey of adults only, with no dietary information, was collected in 1974-1975. Before medical examinations were performed, trained interviewers visited the subjects in their homes to administer socioeconomic and medical history questionnaires. A detailed sub-sample of 3,478 subjects aged 30 or older was given a more detailed medical history questionnaire and a spirometric examination. The detailed subsample was also representative of the U.S. population. This included standard respiratory questions from the National Heart, Lung, and Blood Institute questionnaire on chronic respiratory disease. They also included smoking history, including whether 
the subject had ever smoked one hundred cigarettes, current number of cigarettes smoked per day, maximal cigarettes per day, years of smoking, and years since quitting. A Food Frequency questionnaire asked about usual consumption of fish in the past three months. Intake of fish was coded as portions per week. Interviewers and medical examiners who conducted the NHANES I survey were specially trained to ensure that conduct of the survey at each site was standardized.

Spirometric examinations were performed using an Ohio Medical Instruments Corporation Model 800 electronic spirometer, a storage $\mathrm{x}-\mathrm{y}$ oscilloscope that displayed flow-volume curves for monitoring purposes, a single channel linear script chart recorder, and a data acquisition unit to encode, convert, and record on digital tape the spirometry signals. A pneumatic 5 litre calibration procedure was performed at the beginning of each session on each day. The electronic portion of the instrumentation was calibrated before each subject was tested. Barometric pressure, temperature, saturated (BPTS) correction factors were computed and encoded. Further details have been published elsewhere [3].

All subjects were given a complete physical examination by a physician before they performed the spirometric examination. Subjects who were pregnant, or had acute or chronic medical conditions that the physician felt made a spirometry manoeuvre inappropriate were excluded from the spirometry examination. At least five trials were required for a test set to be complete for an individual. The best trial was defined as the trial with the maximum sum of forced vital capacity FVC and forced expiratory volume in one second $\left(\mathrm{FEV}_{1}\right)$ from at least two reproducible and error free trials. If reproducibility was not achieved the subjects were excluded from the analysis. Further details have been published [3]. The data from the NHANES I were compared to results from the Harvard Six City Study, and, adjusting for height and age, the results were essentially identical [3]. These exclusions reduced the potential sample size from 3,478 to 2,526 .

As noted previously, the variance of the pulmonary function measurements increases with height and age [3]. Logarithmic transformation of the pulmonary function measurements achieved homoscedastic residuals with respect to height and age. Hence regression analyses were done using $\log \left(\mathrm{FEV}_{1}\right)$ as the dependent variable. The units of the dependent variable are the log of the $\mathrm{ml}$ of $\mathrm{FEV}_{1}$. Age and height were also converted to their natural logarithm transformation in the analysis. In a regression of the logarithm of lung function against an explanatory variable, the coefficient of the variable is easily related to the percent difference from predicted by the equation:

$$
100 \times(\exp (\beta)-1)=\% \text { difference from predicted }
$$

for a unit change in the explanatory variable.

Cigarette smoking is clearly an important predictor of $\mathrm{FEV}_{1}$. To assure our assessment of dietary fish intake was not confounded by any correlation with smoking, we first developed the best models we could using smoking variables, before examining fish intake. Current cigarettes per day were used to index the effect of current smoking. We note that a model for the logarithm of $\mathrm{FEV}_{1}$ is a multiplicative model, so the distinction between main effects and interactions is less clear. Lung function may not decrease linearly with cigarettes per day, so a square root transform of cigarettes per day was also considered as a variable in the models. Such a transformation has proved superior to the untransformed variable in evaluating the association of cigarette smoking with markers of inflammation such as peripheral white cell counts [4].

Cumulative smoking may be described either by pack years or by years smoked. We considered both possibilities. Again, square root transforms of pack years and years smoked were also considered. A dummy variable for former smokers was also examined. The different combinations of terms for current and past smoking were examined, and the terms that best fit the model were chosen. Interaction terms were included only if at least marginally significant $(\mathrm{p}<0.10)$. Once the smoking model was developed, we considered the impact of dietary fish intake on $\mathrm{FEV}_{1}$. Dietary fish intake is strongly associated with socioeconomic status. To test whether that might be confounding any observed association, we reestimated our regression controlling for family income. This reduced the sample size by 103 individuals who did not provide such data. We also examined whether the impact of fish consumption varied by age or gender. To avoid a major loss of power, this was done by testing interaction terms for fish consumption by females, and for fish consumption in persons over the age of $52 \mathrm{yrs}$.

The NHANES I survey was a stratified clustered sample, with all the subjects being drawn from the 90 geographic sites. Subjects who live in the same area are often more alike on certain characteristics than subjects randomly chosen from the population. If those characteristics are important for the outcomes being studied, and are not perfectly controlled for, there is likely to be correlation between the residuals from subjects within each site. We have dealt with these design effects by incorporating a random site effect into our analysis. In addition, we have estimated robust variance estimates. This method gives standard errors for the regression coefficients that are asymptotically unbiased even if the correlation in the residuals is not perfectly specified. To estimate these random site effects and robust variances, we have used the Generalized Estimating Equations of ZEGER and LIANG [5]. The GEE macro of M.R. Karim of the Department of Biostatistics, Johns Hopkins School of Hygiene and Public Health was used.

\section{Results}

The distribution of variables used in this analysis is shown in table 1 . Table 2 compares subjects without spirometry to those with spirometry values. Subjects without spirometry values were more likely to have asthma or bronchitis, more likely to be a smoker, and had greater pack yrs of exposure. The square root transform of cigarettes per day was a better predictor of $\mathrm{FEV}_{1}$ than a linear form. The baseline model is shown in table 3 . 
Table 1. - Distribution of continuous variables used in the analysis of NHANES I spirometry

\begin{tabular}{lcccccc}
\hline Percentile & $10 \%$ & $25 \%$ & $50 \%$ & $75 \%$ & $90 \%$ & Mean \\
\hline Age yrs & 35 & 43 & 53 & 63 & 69 & 52.5 \\
Cigarettes/day & 0 & 0 & 0 & 10 & 20 & 6.9 \\
Pack yrs & 0 & 0 & 5 & 33 & 57 & 20.3 \\
FEV ml & 1664 & 2111 & 2632 & 3207 & 3834 & 2687 \\
Height cm & 156 & 160 & 167 & 174 & 180 & 167 \\
Body Mass Index & 20.6 & 22.6 & 25.3 & 28.6 & 32.0 & 26.0 \\
$\quad$ kg.m-2 & & & & & & 1 \\
Fish intake portions/wk & 0.5 & 0.5 & 1 & 1 & 2 & 1 \\
\hline
\end{tabular}

Table 2. - Means of selected variables among persons included in the analysis, and persons excluded because of missing $\mathrm{FEV}_{1}$ measurements

\begin{tabular}{lccc}
\hline & Included & Excluded & $\mathrm{p}$ \\
\hline Smoker \% & 35 & 39 & 0.017 \\
Cigarettes/day & 6.91 & 7.25 & $0.101^{*}$ \\
Pack yrs & 20.3 & 23.1 & $0.021^{*}$ \\
Bronchitis \% & 5.0 & 8.0 & 0.001 \\
Asthma \% & 2.9 & 5.1 & 0.007 \\
Working \% & 56 & 45 & 0.001 \\
Age yrs & 52.5 & 54.5 & $0.110^{*}$ \\
Fish intake & 1.02 & 1.03 & $0.810^{*}$ \\
$\quad$ portions/wk & & & \\
Female \% & 53 & 50 & 0.16 \\
\hline
\end{tabular}

*From Mantel Haenszel statistic, others from chi square statistic.

Table 3. - Regression coefficients for baseline model for $\mathrm{FEV}_{1}$ (natural logarithm) in the First National Health and Nutrition Examination Survey (NHANES I)

\begin{tabular}{|c|c|c|c|}
\hline & $\beta$ & S.E. (robust) & $\mathrm{p}$ \\
\hline Intercept & -8.51 & 1.03 & 0.0001 \\
\hline Ln (age) & 0.676 & 0.589 & 0.250 \\
\hline$[\text { Ln (age) }]^{2}$ & -0.148 & 0.078 & 0.056 \\
\hline Ln (height) & 2.20 & 0.182 & 0.0001 \\
\hline Body Mass Index $\mathrm{kg} \cdot \mathrm{m}^{2}$ & -0.0021 & 0.0009 & 0.257 \\
\hline White* & 0.156 & 0.014 & 0.0001 \\
\hline Sex** & -0.116 & 0.021 & 0.0001 \\
\hline Pack yrs & -0.0011 & 0.0004 & 0.0001 \\
\hline Cigarettes/day & -0.0089 & 0.003 & 0.0051 \\
\hline Working ${ }^{+}$ & 0.051 & 0.018 & 0.0037 \\
\hline
\end{tabular}

*: $1=$ white, $2=$ non-white; $* *$ : male $=1$; female $=2 ;{ }^{\dagger}$ : square root transform; ${ }^{+}$: $1=$ working, $0=$ not working; S.E. standard error.

When added to the baseline model dietary fish intake showed a protective association with $\mathrm{FEV}_{1}(\beta=0.0088 \pm$ $0.0040, \mathrm{p}=0.028)$. When current asthmatic or bronchitic subjects were excluded, the impact of dietary fish intake did not change significantly $(\beta=0.0080 \pm 0.0042$, $\mathrm{p}=0.059)$. When smokers were excluded, the effect of fish intake appeared to increase slightly $(\beta=0.0108 \pm 0.0058$, $\mathrm{p}=0.061$ ). When fish intake was divided into categories, a stepped dose-response relationship was observed. Compared to subjects who consumed fewer than one portion of fish per week, subjects who consumed one portion per week had a covariate adjusted $1.35 \%$ higher $\mathrm{FEV}_{1}(\mathrm{SEM}=1.35 \%)$, and subjects consuming more than one portion per week had a covariate adjusted 2.51 higher $\mathrm{FEV}_{1}(\mathrm{SEM}=1.16 \%)$. The test for trend across the categories was significant $(\mathrm{p}=0.04)$. Controlling for family income had little effect on the association between dietary fish intake and $\mathrm{FEV}_{1}$, except for the reduction in sample size $(\beta=0.0085 \pm 0.0043, p=0.048)$. There was no evidence of a different slope for dietary fish intake in females $(\mathrm{p}=0.46)$ or older subjects $(\mathrm{p}=0.60)$.

\section{Discussion}

In a sample of 2,526 adult subjects aged 30-70 yrs from the First National Health and Nutrition Survey we found a monotonic relationship between dietary portions of fish per week and the natural logorithm of predicted $\mathrm{FEV}_{1}$. The magnitude of the level of effect observed in this study was $115 \mathrm{ml}$ difference in $\mathrm{FEV}_{1}$ between those eating fish less than once a week and those eating fish more than once a week. This relationship was insensitive to the exclusion of currently symptomatic asthmatic or bronchitic subjects. The effect size was slightly larger when smokers were excluded from the analysis. Finally, dietary fish intake did not appear to be a proxy for socioeconomic status.

To our knowledge, no epidemiologic studies have been performed concerning the relationship between dietary fish intake and any respiratory outcome. Short term physiologic studies have yielded conflicting results on the relationship of dietary eicosapentaenoic acid (EPA) and its effect on asthma. KIRSCH and co-workers studied the effect of EPA on 12 adult asthmatic patients [6]. Pulmonary function tests were done at baseline and at $3,6,10,12$ and 14 wks after ingesting either a low dose EPA $\left(0.1 \mathrm{~g} \cdot \mathrm{day}^{-1}\right)$ or high dose EPA $\left(4.0 \mathrm{~g} \cdot \mathrm{day}^{-1}\right)$ for a total of 8 weeks. No significant effect of either high or low dose EPA ingestion on pulmonary function was noted. However, this study did note an alteration, in both the high and the low dose groups, in leukocyte function in patients with asthma. This effect was to decrease the leukocyte content of arachidonic acid and the generation of the leukotriene $\mathrm{B}_{4}\left(\mathrm{LTB}_{4}\right)$ and prostaglandin $\mathrm{E}_{2}\left(\mathrm{PGE}_{2}\right)$ from arachidonic acid. Only the high dose EPA, however, led to significant inhibition of polymorphonuclear leukocyte chemotaxis. No significant effect on symptoms was noted in this investigation. PARM and co-workers studied 20 adult subjects with mild asthma [7]. Twelve subjects received capsules containing $3.2 \mathrm{~g}$ of the EPA and $2.2 \mathrm{~g}$ of docosahexaenoic acid daily, and 8 asthmatic subjects received placebo capsules containing olive oil for $10 \mathrm{wks}$ in a double-blind fashion. Airways responsiveness to histamine, diurnal peak flow variability, symptoms scores and bronchodilator 
use were unchanged by fish oil supplementation. This study confirmed the results of KIRSCH et al. [6] in that neutrophil leukotriene $\mathrm{B}_{4}$ generation was decreased by EPA. In addition, neutrophil chemotaxis was substantially and significantly suppressed.

PAYAN et al. [8] examined two groups of 6 adult asthmatics who were receiving 0.1 or $4 \mathrm{~g}$ of EPA daily for 8 weeks, and also found reduction in leukocyte arachidonic acid and $\mathrm{LTB}_{4}$ and $\mathrm{PGE}_{2}$ with the higher dose of EPA. Although a variety of studies have documented an effect of fish oil on leukotriene production in vivo from inflammatory cells [6-8] our study is the first to examine this issue in an epidemiologic context and find a significant relationship between ordinary dietary fish intake and level of pulmonary function.

We wish to stress some of the methodologic deficiencies of our investigation. This investigation is a cross-sectional study and thus cannot establish a temporal relationship between fish intake and pulmonary function. In addition, food frequency questionnaire data is inherently variable [9]. Also, supplemental use of fish oil and other dietary supplements were not examined. Finally, the effects of medication use on our results cannot be assessed. It is certainly possible that subjects who eat fish on a regular basis are those who follow a more healthy lifestyle and it is not the fish per se but some other dietary component correlated with fish which is responsible for these associations. However, further evaluation of fish intake and its relationship to pulmonary function and asthma symptoms in epidemiology would be worthwhile.

\section{References}

1. Horrobin DF. Low prevalences of coronary heart disease (CHD), psoriasis, asthma and rheumatoid arthritis in Eskimos: Are they caused by high dietary of eicosapentaenoic acid (EPA), genetic variation of essential fatty acid (EFA) metabolism or a combination of both? Medical Hypotheses 1987; 22: 421-428.

2. Plan and Operation of the Health and Nutrition Examination Survey. DHEW Publication (PHS) 799-1310. National Center for Health Statistics, 1965.

3. Chestnut LG, Schwartz J, Savitz DA, Burchfiel CM. Pulmonary function and ambient particulate matter: Epidemiological evidence from NHANES I. Arch Environ Health 1991; 46: 135-144.

4. Schwartz J, Weiss ST. Environmental factors influencing the peripheral blood leukocyte count. Am J Epi 1991; 134: 1402-1409.

5. Zeger SL, Liang K-Y. Longitudinal data analysis for discrete and continuous outcomes. Biometrics 1986; 42: $121-130$.

6. Kirsch CM, Payan DG, Wong MYS, et al. Effect of eicosapentaenoic acid in asthma. Clin Allergy 1988; 18: 177-187.

7. Parm J, Horton CE, Mencia-Huerta JM, et al. Effect of dietary supplementation with fish oil lipids in mild asthma. Thorax 1988; 43: 84-92.

8. Payan DG, Wong MYS, Chernov-Rogan T, et al. Alterations in human leukocytes function induced by ingestion of eicosapentaenoic acid. J Clin Immunol 1986; 6: 402-410.

9. Willett W. Nutritional Epidemiology, N.Y. Oxford University Press, pp. 1-396. 